\title{
Monitoring for adverse events among patients on tuberculosis therapy
}

\section{David Michail}

Department of Respiratory and Sleep Medicine, Westmead Hospital and Blacktown Hospital

Parramatta Chest Clinic

Tuberculosis (TB) continues to be a significant public health problem in New South Wales (NSW) with 512 case notifications in 2011. ${ }^{1}$ The NSW Health Policy Directive on the management of people with TB in NSW states:

The aim of the NSW Department of Health TB Program is to minimise the burden of $T B$ and to prevent the transmission of TB through early detection and appropriate treatment of people with TB. Patients with suspected TB should be referred for management in an Area Health Service (AHS) TB Prevention and Control Service - Chest Clinic. ${ }^{2}$

In Australia, patients with diagnosed or clinically suspected TB are treated under the guidance of a specialist with training in TB management. Standard therapy for TB follows international and Australian guidelines and traditionally involves an intensive phase of drug therapy followed by a continuation phase. The majority of treatment - with only rare exceptions - is administered by directly observed therapy (DOT) to aid with compliance and completion of therapy. ${ }^{2-4}$ The overall goals of treatment are to cure the patient and to minimise the risk of transmission to others.

Side effects and adverse reactions to anti-TB therapy are not uncommon and can be serious and even lifethreatening when they do occur. While various definitions of adverse drug reactions (ADRs) exist a comprehensive definition is:

An appreciably harmful or unpleasant reaction, resulting from an intervention related to the use of a medicinal product, which predicts hazard from future administration and warrants prevention or specific treatment, or alteration of the dosage regimen, or withdrawal of the product. ${ }^{5}$

Most medications used in standard anti-TB regimens were developed decades ago, and standard treatment regimens carry a risk of up to $30 \%$ for significant ADRs developing in the intensive phase of treatment. ${ }^{6}$ Other studies have reported fewer incidences. ${ }^{7-9}$ A significant ADR is one that necessitates interruption of therapy. The most commonly noted include hepatotoxicity and fulminant liver failure, peripheral neuropathy, rash, and visual disturbance, amongst others. ${ }^{6}$ Systems aimed at rapid identification of potential ADRs may be able to minimise their impact on morbidity and mortality and improve tolerance and successful completion of anti-TB therapy.

Early identification of ADRs that occur during the course of therapy for TB requires:

- a thorough knowledge of reported adverse reactions associated with specific drugs

- awareness of clinical situations, including co-morbidity and co-administration of other drugs, that increase a given individual's risk ${ }^{10}$ (individuals who have HIV co-infection and are on antiretroviral therapy are one important high-risk group)

- careful surveillance for the early manifestations of ADRs (which, in turn, requires vigilance on the part of both patients and health care workers).

Although there are no standard guidelines for surveillance regimens, there is widespread agreement on some elements that are standard practice at the Parramatta Chest Clinic, Sydney, Australia, which provides services for Western Sydney residents, including patients of Westmead and Blacktown Hospitals.

\section{General principles of monitoring}

- Appropriate prescriber and health care provider education regarding common and less common side effects of frequently prescribed TB medications.

- Thorough baseline assessment of individual risk, including appropriate blood tests, and review of co-morbidities, medication and lifestyle factors.

- Detailed patient education and pre-treatment counselling, including written information (in the patient's native language where possible) regarding symptoms or signs to monitor, and appropriate health care provider contact details and/or management plan in case of development of symptoms or signs.

- Checklist of symptoms to be completed at interaction between health care provider and patient during therapy administration at DOT visits. The practice of DOT gives a scheduled frequent interaction between the health care provider and the patient that should facilitate frequent symptom review and early identification of any potential problems.

- Periodic TB specialist consultation for symptom review and examination. 
- Appropriate use of repeat blood testing, either scheduled or for further assessment of symptoms. ${ }^{11}$

- Periodic ophthalmic review either through a local Eye Clinic or by appropriately trained TB Clinic staff.

Management of ADRs during therapy for TB requires specialist knowledge. It frequently involves discontinuation of one or all TB medications, as well as supportive management until the ADR has resolved. Decisions on the timely reintroduction of appropriate therapy and the need to increase the duration of therapy to compensate for treatment interruptions are challenges that face treating physicians.

\section{Management of adverse drug reactions}

Hepatotoxicity is a particularly concerning and difficult to manage complication of TB therapy. Any one of, or a combination of, isoniazid, rifampicin and pyrazinamide (three of the four first-line drugs) may cause hepatotoxicity.

Hepatotoxicity may be identified as a result of presentation with abnormal symptoms and/or signs (anorexia, nausea, vomiting, abdominal discomfort, right upper quadrant tenderness or jaundice) or by routine surveillance blood tests. Management practice at the Parramatta Chest Clinic varies between the following two scenarios: ${ }^{12-14}$

- Asymptomatic patients with an increase in serum transaminases from baseline - if the increase in transaminases is $<5$-fold over normal baseline value, continue the current regimen, monitor for development of symptoms of liver dysfunction, and continue to periodically check liver function tests (minimum recommended frequency at least weekly). If the transaminases increase $>5$-fold over normal baseline normal value, cease all medications

- Symptomatic patients - withhold all drugs and measure liver function and coagulation status immediately. If transaminases are elevated $>3$-fold over normal baseline values, continue to withhold all drugs until symptoms resolve and transaminases decrease to $<2$-fold above normal, then re-introduce medications under specialist guidance.

For other severe ADRs, the following practices are used at the Parramatta Chest Clinic:

- full assessment of the patient by a member of the Chest Clinic or Respiratory Department medical/nursing staff as soon as possible, including comprehensive examination and blood testing

- immediate cessation of all anti-TB drugs

- review of other non-TB medications that may be contributing, or medications requiring dose adjustment in liver failure (if the drug reaction has caused hepatotoxicity)

- supportive care as required

- consideration of admission to hospital if indicated.
Once a patient has recovered from the drug reaction, reintroduction of therapy is undertaken under the guidance of a TB specialist.

\section{Conclusion}

TB remains a significant public health problem in NSW. With rapid identification of active cases and appropriate therapy under the guidance of a team well trained in the management of TB patients, successful therapy with minimisation of risk of adverse reactions can be achieved. Thorough knowledge of potential symptoms and signs of drug reactions, as well as frequent nurse and physicianinitiated surveillance, are key.

\section{References}

1. Lowbridge C, Christensen AJ, McAnulty JM. EpiReview: Tuberculosis in NSW, 2009-2011. N S W Public Health Bull 2013; 24(1): 3-9.

2. NSW Health. Tuberculosis - Principles for Management of people with Tuberculosis in NSW. Policy Directive PD2008_019. Available at: http://www0.health.nsw.gov.au/ policies/pd/2008/pdf/PD2008_019.pdf(Cited 4 February 2013).

3. Tekle B, Mariam DH, Ali A. Defaulting from DOTS and its determinants in three districts of Arsizone in Ethiopia. Int $J$ Tuberc Lung Dis 2002; 6: 573-9.

4. Fry RS, Khoshnood K, Vdovichenko E, Granskaya J, Sazhin V, Shpakovskaya L et al. Barriers to completion of tuberculosis treatment among prisoners in St. Petersburg, Russia. Int J Tuberc Lung Dis 2005; 9: 1027-33.

5. Edwards IR, Aronson JK. Adverse drug reactions: definitions, diagnosis, and management. Lancet 2000; 356: 1255-9. doi:10.1016/S0140-6736(00)02799-9

6. Marra F, Marra CA, Bruchet N, Richardson K, Moadebi S, Elwood RK et al. Adverse drug reactions associated with firstline anti-tuberculosis drug regimens. Int J Tuberc Lung Dis 2007; 11(8): 868-75.

7. Schaberg T, Rebham K, Lode H. Risk factors for side-effects of isoniazid, rifampicin and pyrazinamide in patients hospitalized for pulmonary tuberculosis. Eur Respir J 1996; 9: 2026-30. doi:10.1183/09031936.96.09102026

8. Zierski M, Bek E. Side-effects of drug regimens used in shortcourse regimens chemotherapy for pulmonary tuberculosis. A controlled clinical study. Tubercle 1980; 61(1): 41-9. doi:10.1016/0041-3879(80)90060-4

9. Yee D, Valiquette C, Pelletier M, Parisien I, Rocher I, Menzies D. Incidence of serious side effects from first-line antituberculosis drugs among patients treated for active tuberculosis. Am J Respir Crit Care Med 2003; 167(11): 1472-7. doi:10.1164/rccm.200206-626OC

10. Sharma SK, Balamurugan A, Saha PK, Pandey RM, Mehra NK. Evaluation of clinical and immunogenetic risk factors for the development of hepatotoxicity during anti-tuberculosis treatment. Am J Respir Crit Care Med 2002; 166: 916-9. doi:10.1164/rccm.2108091

11. Durand F, Jebrak G, Pessayre D, Fournier M, Bernuau J. Hepatotoxcity of antitubercular drugs. Rationale for monitoring 
liver status. Drug Saf 1996; 15(6): 394-405. doi:10.2165/ 00002018-199615060-00004

12. Blumberg HM, Burman WJ, Chaisson RE, Daley CL, Etkind SC, Friedman LN et al. American Thoracic Society/Centers for Disease Control and Prevention/Infectious Diseases Society of America: Treatment of tuberculosis. Am J Respir Crit Care Med 2003; 167(4): 603-62. doi:10.1164/rccm.167.4.603
13. Saukkonen JJ, Cohn DL, Jasmer RM, Schenker S, Jereb JA, Nolan CM et al. An official ATS statement: hepatotoxicity of anti-tuberculous therapy. Am J Respir Crit Care Med 2006; 174 : 935-52. doi:10.1164/rccm.200510-1666ST

14. Hopewell PC, Pai M, Maher D, Uplekar M, Raviglione MC. International standards for tuberculosis care. Lancet Infect Dis 2006; 6(11): 710-25. doi:10.1016/S1473-3099(06)70628-4 